\title{
Investigations on the charmless decays of $Y(4260)^{*}$
}

\author{
LI Gang ${ }^{1,3 ; 1)}$ AN Chun-Sheng ${ }^{2 ; 2} \quad$ LI Peng-Yu ${ }^{1}$ \\ $\mathrm{LIU} \mathrm{Di}^{1} \quad \mathrm{ZHANG} \mathrm{Xiao}^{1} \quad \mathrm{ZHOU} \mathrm{Zhu^{1 }}$ \\ ${ }^{1}$ Department of Physics, Qufu Normal University, Qufu 273165, China \\ ${ }^{2}$ Institute of High Energy Physics, and Theoretical Physics Center for Science Facilities, Chinese Academy of Sciences, Beijing 100049, \\ China \\ ${ }^{3}$ State Key Laboratory of Theoretical Physics, Institute of Theoretical Physics, Chinese Academy of Sciences, Beijing 100190, China
}

\begin{abstract}
Apart from the charmful decay channels of $Y(4260)$, the charmless decay channels of $Y(4260)$ also provide us a good platform to study the nature and the decay mechanism of $Y(4260)$. In this paper, we propose to probe the structure of $Y(4260)$ through the charmless decays $Y(4260) \rightarrow V P$ via intermediate $D_{1} \bar{D}+$ c.c. meson loops, where $V$ and $P$ stand for light vector and pseudoscalar mesons, respectively. Under the molecule ansatz of $Y(4260)$, the predicted total branching ratio $B R_{V P}$ for all $Y(4260) \rightarrow V P$ processes are about $\left(0.34_{-0.23}^{+0.32}\right) \%$ to $\left(0.75_{-0.52}^{+0.72}\right) \%$ with the cutoff parameter $\alpha=2 \sim 3$. Numerical results show that the intermediate $D_{1} \bar{D}+$ c.c. meson loops may be a possible transition mechanism in the $Y(4260) \rightarrow V P$ decays. These predicted branching ratios are the same order to that of $Y(4260) \rightarrow Z_{c}^{+}(3900) \pi^{-}$, which may be an evidence of $D_{1} D$ molecule and can be examined by the forthcoming BESIII data in the near future.
\end{abstract}

Key words: Intermediate meson loop, exotic states

PACS: $13.25 . \mathrm{GV}, 13.75 . \mathrm{Lb}, 14.40 . \mathrm{Pq}$

\section{Introduction}

In the past decade, many new charmonium (or charmoniumlike), i.e., the so-called $X Y Z$ states have been observed experimentally, which triggered a lot of theoretical investigations on the nature of exotic meson resonances beyond the conventional $q \bar{q}$ quark model 1 6]. Among these observed $X Y Z$ states, the resonance $Y(4260)$, which was firstly observed by the BaBar Collaboration in the $\pi^{+} \pi^{-} J / \psi$ invariant spectrum in $e^{+} e^{-} \rightarrow \gamma_{I S R} \pi^{+} \pi^{-} J / \psi$ 7], and then confirmed by both the CLEO and Belle Collaborations [8, 9], is a very interesting one because of that its mass $m=4263_{-9}^{+8} \mathrm{MeV}$ [10] is only about $30-40 \mathrm{MeV}$ below the $S$-wave $D_{1} \bar{D}+$ c.c. threshold. And very recently, the new datum from BESIII confirms the signal in $Y(4260) \rightarrow J / \psi \pi^{+} \pi^{-}$with much higher statistics [11]. It indicates that it's worth to study the structure and decays of $Y(4260)$.

Since the observation of $Y(4260)$, many different solutions were proposed to study the structure of $Y(4260)$. These solutions include the $4 S$ charmonium [12], tetraquark $c \bar{c} s \bar{s}$ state [13], charmonium hybrid 14 16], $D_{1} \bar{D}$ molecule $17-19$, ${ }^{*} \chi_{c 1} \omega$ molecule 23, $\chi_{c 1} \rho$ molecule 24], hadrocharmonium state [4, 25, 26], spin-triplet $\Lambda_{c}-\bar{\Lambda}_{c}$ baryonium states 27-30], a cusp 31, 32] or a non-resonance explanation [33, 34] etc. Under the $D_{1} \bar{D}$ molecule ansatz, some experimental observations can be described, such as the observation of $Z_{c}(3900)$ in $e^{+} e^{-} \rightarrow \pi^{+} \pi^{-} J / \psi$ [19], the production of $X(3872)$ in the $e^{+} e^{-}$annihilation around the mass of $Y(4260)$ [20], and the threshold behavior in the main decay channels of $Y(4260)$ [35] etc. In Ref. 26], Li and Voloshin argue that the hadrocharmonium interpretation of $Y(4260)$ may be more credible. Their argument is based on the fact that the production of an $S$-wave pairs with $S_{L}^{P}=(3 / 2)^{+}$and $S_{L}^{P}=(1 / 2)^{-}$heavy mesons, where $S_{L}$ is the sum of the spin of the light quark and the orbital angular momentum in the heavy mesons, in $e^{+} e^{-}$ collisions is forbidden in the limit of exact heavy quark spin symmetry. In Ref. [26], it was also shown that both

Received

* Supported by National Natural Science Foundation of China (11275113,11205164)

1) E-mail: gli@mail.qfnu.edu.cn

2) E-mail: ancs@ihep.ac.cn

(c)2013 Chinese Physical Society and the Institute of High Energy Physics of the Chinese Academy of Sciences and the Institute of Modern Physics of the Chinese Academy of Sciences and IOP Publishing Ltd

* There are two $D_{1}$ states of similar masses, and the one in question should be the narrower one, i.e., the $D_{1}(2420)(\Gamma=27$ $\mathrm{MeV})$, the $D_{1}(2430)(\Gamma \simeq 384 \mathrm{MeV})$ is too broad to form a molecular state $20-22]$. 
the rescattering due to the process $D^{*} \bar{D}^{*} \rightarrow D_{1} \bar{D}$ and the mixing of the $D_{1}(2420)$ with the $D_{1}(2430)$ cannot evade this suppressed production. They also considered the possible kinematic effects that might increase the amount of the heavy quark spin symmetry (HQSS) violation and found that the kinematical effect is quite small at such energy. Thus, they concluded that the $S$-wave $D_{1} \bar{D}$ production is suppressed. In Ref. [36], Wang et al. confront both the hadronic molecule and the hadrocharmonium interpretations of the $Y(4260)$ with the experimental data currently available. Although the production of $(3 / 2)^{+}$and $(1 / 2)^{-}$heavy meson pairs is suppressed in the heavy quark limit [26], the heavy quark spin symmetry breaking effects in the charm sector can be significant. So the resulting suppression for the physical charm quark mass is not in conflict with the interpretation that the main component of the $Y(4260)$ is a $D_{1} \bar{D}$ molecule.

On the other hand, the intermediate meson loop transition as an important nonperturbative dynamical mechanism has been extensively studied in the energy region of charmonium 37 64]. It is widely recognized that the intermediate meson loops may be closely related to some nonperturbative phenomena observed in experiments 46 67], e.g. sizeable branching ratios for non- $D \bar{D}$ decay of $\psi(3770)$ [46 52], the helicity selection rule violations in charmonium decays 59 61], isospin symmetry breaking in charmonium decays [63, 64]. Recently, this intermediate meson loops mechanism has been applied to the production and decays of ordinary and exotic states [19, 20, 35, 68 73].

Recently, the charmful decay channels have been extensively used to constrain the reaction mechanism and gain insights into the nature $Y(4260)$ [35, 68 70]. Apart from the charmful decay channels of $Y$ (4260), the charmless decay channels of $Y(4260)$ are also a good platform to further study $Y(4260)$. In the present work, we study the charmless decays $Y(4260) \rightarrow V P$ via $D_{1} \bar{D}$ loop with an effective Lagrangian approach (ELA) under the $D_{1} \bar{D}+$ c.c. molecule ansatz. The paper is organized as follows. In Sec. 2, we will briefly introduce the ELA and give some relevant formulae, the numerical results are presented in Sec. 3, and Sec. 4 contains a brief summary.

\section{The Model}

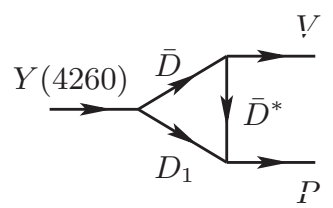

Fig. 1. The hadron-level diagrams for $Y(4260) \rightarrow$ $V P$ with $D_{1} \bar{D}$ as the intermediate states. $V$ and $P$ denote the light vector and pseudoscalar mesons, respectively.
Generally speaking, all the possible intermediate meson exchange loops should be included in the calculation. In reality, the breakdown of the local quark-hadron duality allows us to pick up the leading contributions as a reasonable approximation [74, 75]. For example, the intermediate states involving flavor changes turn out to be strongly suppressed. One reason is because of the large virtualities involved in the light meson loops. The other is because of the Okubo-Zweig-Iizuka-rule suppressions. In this work, we have assumed that $Y(4260)$ is dominated by the $S$-wave $D_{1} \bar{D}+$ c.c. component and the $D_{1} \bar{D}+$ c.c. mass threshold is only $30 \mathrm{MeV}$ above the $Y(4260)$, so we consider the $\mathrm{S}$-wave $D_{1} \bar{D}$ meson loops as the leading contributions.

By assuming $Y(4260)$ is an S-wave $D_{1} \bar{D}$ molecular state, the effective Lagrangian is constructed as

$$
\mathcal{L}_{Y(4260) D_{1} D}=i \frac{x}{\sqrt{2}}\left(\bar{D}_{a}^{\dagger} Y^{\mu} D_{1 a}^{\mu \dagger}-\bar{D}_{1 a}^{\mu \dagger} Y^{\mu} D_{a}^{\dagger}\right)+H . c .,
$$

where $x$ is the coupling constant.

For a state slightly below an S-wave two-hadron threshold, the effective coupling constant of this state to the two-body channel, $g_{N R}$, is related to the probability of finding the two-hadron component in the physical wave function of the bound state, $c^{2}$, and the binding energy, $\epsilon=m_{1}+m_{2}-M[20,76,77]$

$$
g_{\mathrm{NR}}^{2} \equiv 16 \pi\left(m_{1}+m_{2}\right)^{2} c^{2} \sqrt{\frac{2 \epsilon}{\mu}}[1+\mathcal{O}(\sqrt{2 \mu \epsilon r})],
$$

where $\mu=m_{1} m_{2} /\left(m_{1}+m_{2}\right)$ is the reduced mass, and $r$ denotes the range of the forces. Notice that the coupling constant gets maximized for a pure bound state, which has $c^{2}=1$ by definition.

Using the masses of the $Y(4260), D$ and $D_{1}$ given in PDG [10], we obtain the mass difference between the $Y(4260)$ and the $D_{1} \bar{D}+$ c.c. threshold to be $m_{D}+m_{D_{1}}-$ $m_{Y}=27_{-8}^{+9} \mathrm{MeV}$. Assuming that $Y(4260)$ is pure $D D_{1}$ molecule, which corresponding to hte probability of finding $D_{1} \bar{D}$ component in the physical wave function of the bound states $c^{2}=1$, we obtain the coupling constant $x$

$$
|x|=14.62_{-1.25}^{+1.11} \pm 6.20 \mathrm{GeV},
$$

where the first errors are due to the uncertainties of the binding energies, and the second ones are from the the approximate nature of Eq. (2).

The effective Lagrangian relevant to the light vector mesons can be obtained as follows [78, 79],

$$
\begin{aligned}
\mathcal{L}_{\mathcal{V}}= & i g_{\mathcal{D}^{*} \mathcal{D} \mathcal{V}} \epsilon_{\alpha \beta \mu \nu}\left(\mathcal{D} \overleftrightarrow{\partial_{\alpha}} \mathcal{D}^{* \beta \dagger}-\mathcal{D}^{* \beta \dagger} \overleftrightarrow{\partial_{\alpha}} \mathcal{D}^{j}\right) \partial^{\mu} \mathcal{V}^{\nu} \\
& +i g_{\overline{\mathcal{D}}^{*} \overline{\mathcal{D}} \mathcal{V}} \epsilon_{\alpha \beta \mu \nu}\left(\overline{\mathcal{D}} \overleftrightarrow{\partial_{\alpha}} \overline{\mathcal{D}}^{* \beta \dagger}-\overline{\mathcal{D}}^{* \beta \dagger} \overleftrightarrow{\partial_{\alpha}} \overline{\mathcal{D}}^{j}\right) \partial^{\mu} \mathcal{V}^{\nu} \\
& + \text { H.c. }
\end{aligned}
$$

and the effective Lagrangian for the light pseudoscalar mesons are constructed based on both heavy quark spinflavor transformation and chiral transformation [80 83]. 
Accordingly, the interaction terms studied in the present work read

$$
\begin{aligned}
\mathcal{L}_{\mathcal{P}}= & g_{D_{1} \mathcal{D}^{* \mathcal{P}}}\left[3 D_{1}^{\mu}\left(\partial_{\mu} \partial_{\nu} \mathcal{P}\right) \mathcal{D}^{* \dagger \nu}-D_{1}^{\mu}\left(\partial^{\nu} \partial_{\nu} \mathcal{P}\right) \mathcal{D}_{\mu}^{* \dagger}\right] \\
& +g_{\bar{D}_{1} \overline{\mathcal{D}}^{* \mathcal{P}}}\left[3 \overline{\mathcal{D}}^{* \dagger \mu}\left(\partial_{\mu} \partial_{\nu} \mathcal{P}\right) \bar{D}_{1}^{\nu}-\overline{\mathcal{D}}^{* \dagger \mu}\left(\partial^{\nu} \partial_{\nu} \mathcal{P}\right) \bar{D}_{1 \nu}\right] \\
& + \text { H.c. }
\end{aligned}
$$

with $\mathcal{D}^{(*)}=\left(D^{(*)+}, D^{(*) 0}, D_{s}^{(*)+}\right)$ and $\overline{\mathcal{D}}^{(*)}=$ $\left(D^{(*)-}, \bar{D}^{(*) 0}, D_{s}^{(*)-}\right) . \mathcal{P}$ and $\mathcal{V}$ denote the $3 \times 3$ matrices for the pseudoscalar octet and vector nonet, respectively [55], i.e.,

$\mathcal{P}=\left(\begin{array}{ccc}\frac{\pi^{0}}{\sqrt{2}}+\frac{\eta \cos \alpha_{P}+\eta^{\prime} \sin \alpha_{P}}{\sqrt{2}} & \pi^{+} & K^{+} \\ \pi^{-} & -\frac{\pi^{0}}{\sqrt{2}}+\frac{\eta \cos \alpha_{P}+\eta^{\prime} \sin \alpha_{P}}{\sqrt{2}} & K^{0} \\ K^{-} & \bar{K}^{0} & -\eta \sin \alpha_{P}+\eta^{\prime} \cos \alpha_{P}\end{array}\right), \mathcal{V}=\left(\begin{array}{ccc}\frac{\rho^{0}}{\sqrt{2}}+\frac{\omega}{\sqrt{2}} & \rho^{+} & K^{*+} \\ \rho^{-} & -\frac{\rho^{0}}{\sqrt{2}}+\frac{\omega}{\sqrt{2}} & K^{* 0} \\ K^{*-} & \bar{K}^{* 0} & \phi\end{array}\right)$

The physical states $\eta$ and $\eta^{\prime}$, which should be linear combinations of $n \bar{n}=(u \bar{u}+d \bar{d}) / \sqrt{2}$ and $s \bar{s}$, are taken to be the following form

$$
\begin{aligned}
|\eta\rangle & =\cos \alpha_{P}|n \bar{n}\rangle-\sin \alpha_{P}|s \bar{s}\rangle, \\
\left|\eta^{\prime}\right\rangle & =\sin \alpha_{P}|n \bar{n}\rangle+\cos \alpha_{P}|s \bar{s}\rangle,
\end{aligned}
$$

where $\alpha_{P} \simeq \theta_{P}+\arctan \sqrt{2}$. Empirical value for the pseudoscalar mixing angle $\theta_{P}$ should in a range of $-22^{\circ} \sim$ $-13^{\circ}$ [10], and here we take $\theta_{P}=-19.3^{\circ}$ [54].

And the coupling constants relevant to the light vector mesons in Eq. (4) read

$$
g_{\mathcal{D} * \mathcal{D} \mathcal{V}}=-g_{\overline{\mathcal{D}} * \overline{\mathcal{D}} \mathcal{V}}=-\frac{1}{\sqrt{2}} \lambda g_{V},
$$

where $f_{\pi}=132 \mathrm{MeV}$ is the pion decay constant, and the parameter $g_{V}$ is given by $g_{V}=m_{\rho} / f_{\pi}$ [83]. By matching the form factor obtained from the light cone sum rule and that calculated from the Lattice QCD, we can obtain the parameter $\lambda=0.56 \mathrm{GeV}^{-1}[84]$.

In the chiral and heavy quark symmetry limit, the coupling constants relevant to the pseudoscalar mesons in Eq. (5) are

$$
g_{\mathrm{D}^{*} \mathrm{D}_{1} \mathrm{P}}=g_{\overline{\mathrm{D}}^{*} \overline{\mathrm{D}}_{1} \mathrm{P}}=-\frac{\sqrt{6}}{3} \frac{h^{\prime}}{\Lambda_{\chi} f_{\pi}} \sqrt{\mathrm{m}_{\mathrm{D}^{*} \mathrm{~m}_{\mathrm{D}_{1}}}} .
$$

Here $\Lambda_{\chi}$ is the momentum scale characterising the convergence of the derivative expansion, usually taken as the chiral symmetry breaking scale $\Lambda_{\chi} \simeq 1 \mathrm{GeV}$. The coupling $h^{\prime}$, which is relevant to $\Delta_{H}$, i.e., the difference between the charmed meson doublet mass and the mass of the heavy quark involved, can be obtained in a constituent quark-meson model [85]. If one take the value $\Delta_{H}=0.4 \pm 0.1 \mathrm{GeV}$, then one can obtain $h^{\prime}=$ $0.65_{-0.30}^{+0.44}$ 85]. As the total $D_{2}^{* 0}$ width is dominated by the one pion mode in the chiral heavy meson Lagrangian, one can use the experimental result of $49.0 \pm 1.4 \mathrm{MeV}$ to extract an experimental value for $h^{\prime}$ to be $0.74 \pm 0.01$ [10]. Here, we take $h^{\prime}=0.74 \pm 0.01$ as an estimate.

The loop transition amplitudes for the transitions in Fig. 1 can be expressed in a general form in the effective Lagrangian approach as follows,

$$
\mathcal{A}_{f i}=\int \frac{d^{4} q_{2}}{(2 \pi)^{4}} \sum_{D^{*} \text { pol. }} \frac{T_{1} T_{2} T_{3}}{a_{1} a_{2} a_{3}} \mathcal{F}\left(m_{2}, q_{2}^{2}\right),
$$

where $T_{i}$ and $a_{i}=q_{i}^{2}-m_{i}^{2}(i=1,2,3)$ are the vertex functions and the denominators of the intermediate meson propagators, respectively. As mentioned above, the mass of $Y(4260)$ is slightly below the S-wave $D_{1} \bar{D}$ threshold, so the off-shell effects of intermediate $D_{1}$ and $\bar{D}$ should be smaller than that of the exchanged particle. So in order to take care of the off-shell effects of the exchanged particles [37, 86, 87], we adopt a monopole form factor

$$
\mathcal{F}\left(m_{2}, q_{2}^{2}\right) \equiv \frac{\Lambda^{2}-m_{2}^{2}}{\Lambda^{2}-q_{2}^{2}},
$$

with $\Lambda \equiv m_{2}+\alpha \Lambda_{\mathrm{QCD}}$, and the QCD energy scale $\Lambda_{\mathrm{QCD}}=220 \mathrm{MeV}$.

\section{Numerical Results}

Table 1. The predicted branching ratios of $Y(4260)$ decays with different $\alpha$ values. The uncertainties are dominated by the use of Eq. (2). 


\begin{tabular}{cccc}
\hline Final states & No Form factor & $\alpha=2.0$ & Monopole Form Factor \\
& & $\left(1.93_{-6.12}^{+8.58}\right) \times 10^{-4}$ & $\left(1.98_{-1.36}^{+1.91}\right) \times 10^{-3}$ \\
\hline$\rho^{0} \pi^{0}$ & $\left(1.46_{-1.01}^{+1.41}\right) \times 10^{-2}$ & $\left(2.61_{-1.99}^{+2.51}\right) \times 10^{-3}$ & $\left(5.92_{-4.11}^{+5.72}\right) \times 10^{-3}$ \\
$\rho \pi$ & $\left(4.39_{-3.03}^{+4.25}\right) \times 10^{-2}$ & $\left(1.09_{-0.76}^{+1.06}\right) \times 10^{-4}$ & $\left(3.27_{-2.25}^{+3.12}\right) \times 10^{-4}$ \\
$K^{*+} K^{-}+$c.c. & $\left(4.90_{-3.37}^{+4.72}\right) \times 10^{-3}$ & $\left(1.44_{-0.99}^{+1.38}\right) \times 10^{-4}$ & $\left(3.21_{-2.21}^{+3.09}\right) \times 10^{-4}$ \\
$K^{* 0} \bar{K}^{-}+$c.c. & $\left(4.96_{-3.41}^{+4.78}\right) \times 10^{-3}$ & $\left(3.63_{-2.51}^{+3.51}\right) \times 10^{-4}$ & $\left(8.18_{-5}^{+7.88}\right) \times 10^{-4}$ \\
$\omega \eta$ & $\left(1.37_{-0.95}^{+1.33}\right) \times 10^{-2}$ & $\left(3.47_{-2.39}^{+3.35}\right) \times 10^{-5}$ & $\left(8.38_{-5}^{+8.13}\right) \times 10^{-5}$ \\
$\omega \eta^{\prime}$ & $\left(1.25_{-0.86}^{+1.21}\right) \times 10^{-2}$ & $\left(9.48_{-6.52}^{+9.13}\right) \times 10^{-9}$ & $\left(1.96_{-1.35}^{+1.89}\right) \times 10^{-8}$ \\
$\rho \eta$ & $\left(2.93_{-2.01}^{+2.83}\right) \times 10^{-7}$ & $\left(3.27_{-2.15}^{+3.15}\right) \times 10^{-8}$ & $\left(6.52_{-4.41}^{+6.27}\right) \times 10^{-8}$ \\
$\rho \eta^{\prime}$ & $\left(8.18_{-5.62}^{+7.88}\right) \times 10^{-7}$ & $\left(1.44_{-1.30}^{+1.39}\right) \times 10^{-8}$ & $\left(3.09_{-2.13}^{+2.97}\right) \times 10^{-8}$ \\
$\omega \pi^{0}$ & $\left(5.22_{-3.56}^{+5.02}\right) \times 10^{-7}$ & $\left(3.36_{-2.31}^{+3.24}\right) \times 10^{-3}$ & $\left(7.48_{-5.16}^{+7.22}\right) \times 10^{-3}$ \\
Total & $\left(8.03_{-5.52}^{+7.78}\right) \%$ & & \\
\hline
\end{tabular}

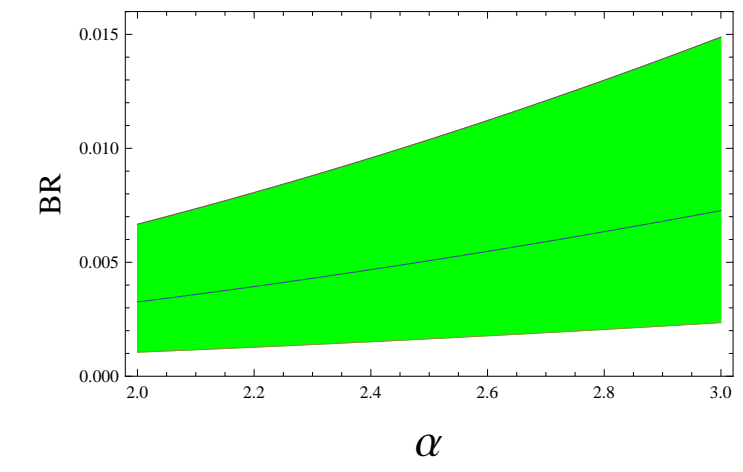

Fig. 2. The $\alpha$ dependence of the total branching ratios of $Y(4260) \rightarrow V P$. The upper and lower limits are obtained with the upper and lower limits of the coupling constant in Eq. (3).

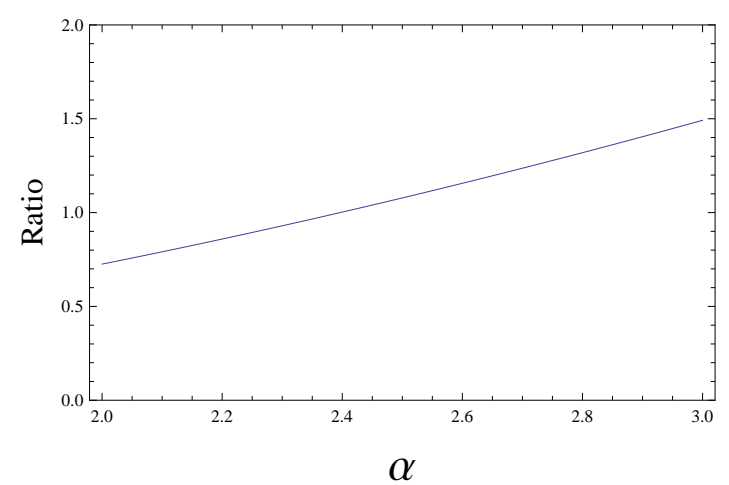

Fig. 3. The $\alpha$ dependence of the total branching ratios of $Y(4260) \rightarrow V P$. The upper and lower limits are obtained with the upper and lower limits of the coupling constant in Eq. (3).

The width of $Y(4260)$ is about $95 \pm 14 \mathrm{MeV}$ [10], so we should take into account the mass distribution of the $Y(4260)$ in the calculations of its decay widths. Then the decay width of $Y(4260) \rightarrow V P$ can be calculated as follow [88],

$$
\begin{aligned}
\Gamma_{Y(4260) \rightarrow V P}= & \frac{1}{W} \int_{\left(m_{Y}-2 \Gamma_{Y}\right)^{2}}^{\left(m_{Y}+2 \Gamma_{Y}\right)^{2}} d s \frac{(2 \pi)^{4}}{2 \sqrt{s}} \\
& \times \int d \Phi_{2}|\mathcal{A}|^{2} \frac{1}{\pi} \operatorname{Im}\left(\frac{-1}{s-m_{Y}^{2}+i m_{Y} \Gamma_{Y}}\right),
\end{aligned}
$$

where $\mathcal{A}$ are the loop transition amplitudes for the processes in Fig. 1. The factor $1 / W$ with

$$
W=\frac{1}{\pi} \int_{\left(m_{Y}-2 \Gamma_{Y}\right)^{2}}^{\left(m_{Y}+2 \Gamma_{Y}\right)^{2}} \operatorname{Im}\left(\frac{-1}{s-m_{Y}^{2}+i m_{Y} \Gamma_{Y}}\right) d s
$$

is used to normalize the spectral function of the $Y(4260)$ state.

Before proceeding to the numerical results, we first discuss the possible uncertainties involved in the calculations. The first uncertainties is the assumption of the probability $c^{2}=1$ for the $D_{1} \bar{D}$ structure for $Y(4260)$. As shown in Eq. (2), the predicted branching ratios are proportional to probability $c^{2}$. The second one comes from the width effects of $Y(4260)$ and the final $\rho$ mesons. We have checked that the width effect of $\rho$ meson only causes a minor change of about $1 \% \sim 5 \%$, which is because the mass of the final states are about $3 \mathrm{GeV}$ below $Y(4260)$.

In Fig. 2, we present the total branching ratio of all possible $Y(4260) \rightarrow V P$ in terms of the cutoff parameter $\alpha$. The upper and lower limits are obtained with the upper and lower limits of the coupling constant in (3). As shown from this figure, there is no cusp structure in the curve. This is because the mass of $Y(4260)$ lies below the intermediate $D_{1} \bar{D}$ threshold. The branching ratios are not drastically sensitive to the cutoff parameter, which indicates a reasonable cutoff of the ultraviolet contributions by the empirical form factors to some extent.

To show the branching ratios of $Y(4260)$ to different $V P$ channels explicitly, we list the predicted branching ratios of $Y(4260)$ for each decay channel with $\alpha=2.0$ and 3.0 in Table. 1, with comparison to the numerical results obtained without a form factor. Notice that the given errors are from the uncertainties of the the coupling constants in Eq. (3). As shown in Table1, the total branching ratio of $Y(4260) \rightarrow V P$ is about $\left(8.03_{-5.52}^{+7.78}\right) \%$ without form factor. Obviously, the obtained branching ratio in this way is somewhat larger than expected. In principle, since the $Y(4260)$ is taken to be a $D_{1} \bar{D}+$ c.c. molecule, so the main decay channel would be $D^{*} \bar{D} \pi$. It is because that the exchanged charmed mesons are 
usually off-shell, which indicates the necessity of considering the form factor. As shown in the last two columns in Table 1 the total branching ratio of $Y(4260) \rightarrow V P$ are from $\left(3.36_{-2.31}^{+3.24}\right) \times 10^{-3}$ to $\left(7.48_{-5.16}^{+7.22}\right) \times 10^{-3}$ with the cutoff parameter $\alpha=2.0 \sim 3.0$.

For the isospin-violating channels, i.e., $Y(4260) \rightarrow$ $\omega \pi^{0}, \rho \eta$, and $\rho \eta^{\prime}$, the charged and neutral charmed meson loops would cancel out exactly in the isospin symmetry limit. In other words, the mass difference between the $u$ and $d$ quark will lead to $m_{\mathcal{D}}^{(*) \pm} \neq m_{\mathcal{D}}^{(*) 0}$ due to the isospin symmetry breaking. As a result, the charged and neutral charmed meson loops cannot completely cancel out, and the residue part will contribute to the isospin-violating amplitudes. The branching ratios of these isospin-violating channels as shown in Table 1 are suppressed. Differing from the isospin-violating channels, since there is no cancelations between the charged and neutral meson loops for the isospin isospin conserved channels, i.e., $Y(4260) \rightarrow \rho \pi, K^{*} \bar{K}+c . c, \omega \eta$, and $\omega \eta^{\prime}$, so the calculated branching ratios of these channels are 3-4 orders of magnitude larger than that of the isospin violated channels. As shown in this table, at the same $\alpha$, the predicted branching ratios of $Y(4260) \rightarrow \omega \eta$ are one order larger than that of $Y(4260) \rightarrow \omega \eta^{\prime}$. The reasons may attribute to the different $n \bar{n}$ component and different phase space. We suggest the experimental measurements to test this point.

In order to better understand the decay mechanism of $Y(4260)$, we define the following ratio

$$
R=\frac{\operatorname{Br}(Y(4260) \rightarrow V P)}{\operatorname{Br}\left(Y(4260) \rightarrow Z_{c}^{+}(3900) \pi^{-}\right)},
$$

which is plotted in Fig. 3 for the dependence on the cutoff parameter. The ratio is less sensitive to the cutoff parameter, which is a consequence of the fact that the involved loops are the same. The predicted branching ratios for $Y(4260) \rightarrow V P$ are the same order to that of $Y(4260) \rightarrow Z_{c}(3900) \pi$. It may be an evidence for the molecule structure of $Y(4260)$ and can be tested by the experimental measurements in future.

\section{Summary}

In this work, we have investigated the charmless decays of $Y(4260)$ in ELA, where $Y(4260)$ is considered as a $D_{1} \bar{D}$ molecular state candidate. We explore the rescattering mechanism with the effective Lagrangian based on the heavy quark symmetry and chiral symmetry. The results show that the $\alpha$ dependence of the branching ratios are not drastically sensitive to some extent. With the commonly accepted $\alpha=2 \sim 3$ range, we make a quantitative prediction for all $Y(4260) \rightarrow V P$ with $B R_{V P}$ from $\left(3.36_{-2.31}^{+3.24}\right) \times 10^{-3}$ to $\left(7.48_{-5.16}^{+7.22}\right) \times 10^{-3}$. These predicted branching ratios are the same order to that of $Y(4260) \rightarrow Z_{c}^{+}(3900) \pi^{-}$with the molecular state assumption. It indicates that the intermediate $D_{1} \bar{D}$ meson loops may be a possible mechanism in $Y(4260) \rightarrow V P$ decays. Of course, the relevant calculations of these $Y(4260) \rightarrow V P$ channels in other models are also needed in order to study the nature of $Y(4260)$ deeply. We expect that with the help of precise measurements of various decay modes at BESIII, the nature of $Y(4260)$ and the decay mechanism of $Y(4260) \rightarrow V P$ can be investigated deeply. And the intermediate meson loops mechanism can be established as a possible nonperturbative dynamics in the charmonium energy region, especially the initial states are close to the two particle thresholds.

\section{References}

1 Brambilla B, Eidelman S, Heltsley B K, Vogt R, Bodwin G T, Eichten E, Frawley A D and Meyer A B et al. Eur. Phys. J. C, 2011, 71: 1534

2 Swanson E S. Phys. Rept., 2006, 429: 243

3 Eichten E, Godfrey S, Mahlke H and Rosner J H. Rev. Mod. Phys., 2008, 80: 1161

4 Voloshin M B. Prog. Part. Nucl. Phys., 2008, 61: 455

5 Godfrey S and Olsen S L. Ann. Rev. Nucl. Part. Sci., 2008, 58: 51

6 Drenska N, Faccini R, Piccinini F, Polosa F A, Renga F and Sabelli C, Riv. Nuovo Cim., 2010, 033: 633

7 Aubert B et al. [BaBar Collaboration]. Phys. Rev. Lett., 2005, 95: 142001

8 HE Q et al. [CLEO Collaboration]. Phys. Rev. D, 2006, 74: 091104

9 YUAN C Z et al. [Belle Collaboration]. Phys. Rev. Lett., 2007, 99: 182004

10 Olive K A et al. [Particle Data Group Collaboration]. Chin. Phys. C, 2014, 38: 1
11 Ablikim $\mathrm{M}$ et al. [BESIII Collaboration]. Phys. Rev. Lett., 2013, 110: 252001

12 Llanes-Estrada F J. Phys. Rev. D, 2005, 72: 031503

13 Maiani L, Riquer V, Piccinini F and Polosa A D. Phys. Rev. D, 2005, 72: 031502

14 ZHU S L. Phys. Lett. B, 2005, 625: 212

15 Kou E and Pene O. Phys. Lett. B, 2005, 631: 164

16 Close F E and Page P R. Phys. Lett. B, 2005, 628: 215

17 DING G J, Zhu J J and YAN M L. Phys. Rev. D, 2008, 77: 014033

18 Ding G J. Phys. Rev. D, 2009, 79: 014001

19 WANG Q, Hanhart C and ZHAO Q. Phys. Rev. Lett., 2013, 111: 132003

20 GUO F K, Hanhart C, Meißner U G, WANG Q and ZHAO Q. Phys. Lett. B, 2013, 725: 127

21 Filin A A, Romanov A, Baru V, Hanhart C, Kalashnikova Y X, Kudryavtsev A E, Meißner U G and Nefediev A V. Phys. Rev. Lett., 2010, 105: 019101

22 GUO F K and Meißner U G. Phys. Rev. D, 2011, 84: 014013

23 YUAN C Z, WANG P and MO X H. Phys. Lett. B, 2006, 634: 399

24 LIU X, ZENG X Q and LI X Q. Phys. Rev. D, 2005, 72: 054023 
25 S. Dubynskiy and Voloshin M B. Phys. Lett. B, 2008, 666: 344

26 LI X and Voloshin M B. Phys. Rev. D, 2013, 588: 034012

27 QIAO C F. Phys. Lett. B, 2006, 639: 263

28 QIAO C F. J. Phys. G, 2008, 35: 075008

29 CHEN Y D and QIAO C F. Phys. Rev. D, 2012, 85: 034034

30 CHEN Y D, QIAO C F, SHEN P. N and ZENG Z Q. Phys. Rev. D, 2013, 88: 114007

31 Beveren E van and Rupp G. arXiv:0904.4351 [hep-ph]

32 Beveren E van and Rupp G. Phys. Rev. D, 2009, 79: 111501

33 Beveren E van, Rupp G and Segovia J. Phys. Rev. Lett., 2010, 105102001

34 CHEN D Y, HE J and LIU X. Phys. Rev. D, 2011, 83054021

35 LIU X H and LI G. Phys. Rev. D, 2013, 88: 014013

36 WANG Q, Cleven M, GUO F K, Hanhart C, Meißner U G, WU X G and ZHAO Q. Phys. Rev. D, 2014, 89: 034001

37 LI X Q, Bugg D V and ZOU B S. Phys. Rev. D, 1997, 55: 1421

38 ZHAO Q and ZOU B S. Phys. Rev. D, 2006, 74: 114025

39 ZHAO Q. Phys. Lett. B, 2006, 636: 197

40 LI G and ZHAO Q. Phys. Rev. D, 2011, 84: 074005

41 LI G, LIU X H and ZHAO Q. Eur. Phys. J. C, 2013, 73: 2576

42 LI G, ZHAO Q and CHANG C H. J. Phys. G, 2008, 35: 055002

43 WANG Q, LI G and ZHAO Q. Phys. Rev. D, 2012, 85: 074015

44 LI G, SHAO F L, ZHAO C W and ZHAO Q. Phys. Rev. D, 2013, 87: 034020

45 LI G and ZHAO Q. Phys. Lett. B, 2008, 670: 55

46 Achasov N N and Kozhevnikov A A. Phys. Lett. B, 1991, 260: 425

47 Achasov N N and Kozhevnikov A A. JETP Lett., 1991, 54: 193 [Pisma Zh. Eksp. Teor. Fiz., 1991, 54: 197]

48 Achasov N N and Kozhevnikov A A. Phys. Rev. D, 1994, 49: 275

49 Achasov N N and Kozhevnikov A A. Phys. Atom. Nucl., 2006, 69: 988

50 ZHANG Y J, LI G and ZHAO Q. Phys. Rev. Lett., 2009, 102: 172001

51 LIU X, ZHANG BO and LI X Q. Phys. Lett. B, 2009, 675: 441

52 LI G, LIU X H, WANG Q and ZHAO Q. Phys. Rev. D, 2013, 88: 014010

53 WU J J, ZHAO Q and ZOU B S. Phys. Rev. D, 2007, 75: 114012

54 LIU X, ZENG X Q and LI X Q. Phys. Rev. D, 2006, 74: 074003

55 CHENG H Y, CHUA C K and Soni A. Phys. Rev. D, 2005, 71: 014030

56 Anisovich V V, Bugg D V, Sarantsev A V and ZOU B S. Phys. Rev. D, 1995, 51: 4619

57 ZHAO Q, ZOU B S and MA Z B. Phys. Lett. B, 2005, 631: 22

58 LI G, ZHAO Q and ZOU B S. Phys. Rev. D, 2008, 77: 014010
59 LIU X H and ZHAO Q. Phys. Rev. D, 2010, 81: 014017

60 WANG Q, LIU X H and ZHAO Q. Phys. Lett. B, 2012, 711: 364

61 LIU X H and ZHAO Q. J. Phys. G, 2011, 38: 035007

62 GUO F K, Hanhart C and Meißner U G. Phys. Rev. Lett., 2009, 103: 082003 [Erratum-ibid., 2010, 104: 109901]

63 GUO F K, Hanhart C, LI G, U. G. Meißner and ZHAO Q. Phys. Rev. D, 2010, 82: 034025

64 GUO F K, Hanhart C, LI G, U. G. Meißner and ZHAO Q. Phys. Rev. D, 2011, 83: 034013

65 LI G, Eur. Phys. J. C, 2013, 73: 2621

66 N. Brambilla et al. [Quarkonium Working Group Collaboration]. hep-ph/0412158

67 Brambilla N, Pineda A, Soto J and Vairo A. Rev. Mod. Phys., 2005, 77: 1423

68 WANG Q, Hanhart C and ZHAO Q. Phys. Lett. B, 2013, 725: 106

69 Cleven M, WANG Q, GUO F K, Hanhart C, Meißner U G and ZHAO Q. arXiv:1310.2190 [hep-ph]

70 WU X G, Hanhart C, WANG Q and ZHAO Q. Phys. Rev. D, 2014, 89: 054038

71 LI G and LIU X H. Phys. Rev. D, 2013, 88: 094008

72 LI G and WANG W. Phys. Lett. B, 2014, 733: 100

73 LI G, LIU X H and ZHOU Z. Phys. Rev. D, 2014, 90: 054006

74 Lipkin H J. Nucl. Phys. B, 1987, 291: 720

75 Lipkin H J. Phys. Lett. B, 1986, 179: 278

76 Weinberg S. Phys. Rev., 1965, 137 B672

77 Baru V et al.. Phys. Lett. B, 2004, 586: 53

78 Casalbuoni R, Deandrea A, Bartolomeo N Di, Gatto R, Feruglio F and Nardulli G. Phys. Lett. B, 1992, 292: 371

79 Casalbuoni R, Deandrea A, Bartolomeo N Di, Gatto R, Feruglio F and Nardulli G. Phys. Lett. B, 1993, 299: 139

80 Burdman G and Donoghue J F. Phys. Lett. B, 1992, 280: 287

81 YAN T M, CHENG H Y, CHEUNG C Y, LIN G L, LIN C Y and YU H L. Phys. Rev. D, 1992,46: 1148 [Erratum-ibid. D, 1997, 55: 5851]

82 Falk A F and Luke M E. Phys. Lett. B, 1992, 292: 119

83 Casalbuoni R, Deandrea A, Bartolomeo N Di, Gatto R, Feruglio F and Nardulli G. Phys. Rept., 1997, 281: 145

84 Isola C, Ladisa M, Nardulli G and Santorelli P. Phys. Rev. D, 2003, 68: 114001

85 Deandrea A, Gatto R, Nardulli G and Polosa A D. JHEP, 1999, 9902: 021

86 Locher M P, LU Y and ZOU B S. Z. Phys. A, 1994, 347: 281

87 LI X Q and ZOU B S. Phys. Lett. B, 1997, 399: 297

88 Cleven M, GUO F K, Hanhart C and Meißner U G. Eur. Phys. J. A, 2011, 47: 120 